increased the odds of symptomatic severe viral infection and death by weakening the immune system or by triggering a hyperactive systemic immune response. The aforementioned publications and concern regarding healthcare personnel risk of acquiring COVID-19 in the surgery setting has led to routine preoperative COVID-19 testing and the delay of nonemergent surgery in many healthcare institutions.

These data raise important questions on timing of nonemergent surgery after asymptomatic and symptomatic COVID-19 infections. With wide availability of COVID-19 RT-PCR testing, many institutions are considering the use of a CDC test-based strategy to remove COVID-19 patients from isolation and clear them for surgery. Complicating this issue is the frequent persistence of positive PCR test results in patients for several weeks. Although infectivity is thought to be low, uncertainty remains, especially in the setting of aerosolizing procedures such as intubation.

The options to clear a recovered COVID-19 patient for surgery could be a symptom or test-based strategy. We recommend a hybrid strategy until more information is available. Surgical procedures should be delayed if possible for 4-6 weeks. We recommend against routine testing in this group as patients are likely not infectious anymore and risk of airway reactivity or ARDS will be reduced. ${ }^{6,8}$ Between 2 and 4 weeks after symptom onset, we recommend a test-based strategy due to insufficient data that recovered COVID patients are completely uninfectious. ${ }^{1,4,5}$ Data from China suggest that patients with severe symptoms have higher viral burden and prolonged viral shedding. ${ }^{1}$ For surgery completion $<2$ weeks after COVID-19 symptoms or diagnosis, we recommend no testing and operating under COVID isolation precautions in the operating room. Immunocompromised patients are likely to shed longer and may be at higher risk of other infectious or pulmonary complications. ${ }^{5}$ We suggest individualized case-based decisions by involved providers until more information is available.

In summary, a hybrid strategy for recovered COVID surgical patients will reinforce the culture of safety in the hospital environment and will reduce excess testing. Our recommendations will be limited in hospital settings where tests are not readily available, test turn-around is slow, or PPE constraints exist. Future studies are needed to examine the duration of infectivity to operating room staff by surgical patients affected by COVID-19, especially when intubation or other aerosolizing procedures are required. As more
COVID-19-positive patients recover, it will also be important to perform postoperative studies related to timing of surgery and risk factors for poor outcomes.

Acknowledgments.

Financial support. No financial support was provided relevant to this article.

Conflicts of interest. All authors report no conflicts of interest relevant to this article.

\section{References}

1. Xiao AT, Tong YI, Zhang S. Profile of RT-PCR for SARS-CoV-2: a preliminary study from 56 COVID-19 patients. Clin Infect Dis 2020 April 19 [Epub ahead of print]. https://doi.org/10.1093/cid/ciaa460.

2. Lan L, Xu D, Guangming Y et al. Positive RT-PCR test results in patients recovered from COVID-19. JAMA 2020;323:1502.

3. Wölfel R, Corman VM, Guggemos W, et al. Virological assessment of hospitalized patients with COVID-2019. Nature 2020;581:465-469.

4. Discontinuation of transmission-based precautions and disposition of patients with COVID-19 in healthcare settings (Interim Guidance). Centers for Disease Control and Prevention website. https://www.cdc. gov/coronavirus/2019-ncov/hcp/disposition-hospitalized-patients.html. Published 2020. Accessed May 20, 2020.

5. Symptom-based strategy to discontinue isolation for persons with COVID-19. Centers for Disease Control and Prevention website. https:// www.cdc.gov/coronavirus/2019-ncov/community/strategy-discontinue-isolation. html. Published 2020. Accessed May 20, 2020.

6. Groeneveld GH, van Paassen J, van Dissel JT, Arbous MS. Influenza season and ARDS after cardiac surgery. N Engl J Med 2018;378:772.

7. Canet J, Gallart L, Gomar C, et al. Prediction of postoperative pulmonary complications in a population-based surgical cohort. Anesthesiology 2010;113:1338.

8. Aquilina AT, Hall WJ, Douglas RG Jr, Utell MJ. Airway reactivity in subjects with viral upper respiratory tract infections: the effects of exercise and cold air. Am Rev Respir Dis 1980;122:3.

9. COVID Surgery Collaborative. Mortality and pulmonary complications in patients undergoing surgery with perioperative SARS-CoV-2 infection: an international cohort study. Lancet 2020 May 29 [Epub ahead of print]. doi: 10.1016/S0140-6736(20)31182-X.

10. Lei S, Jiang F, Su W, et al. Clinical characteristics and outcomes of patients undergoing surgeries during the incubation period of COVID-19 infection. EClinicalMedicine 2020 April 5 [Epub ahead of print]. doi: 10.1016/j.eclinm. 2020.100331 .

\title{
Forewarned but not forearmed: The risk of science driven by immediacy
}

\author{
Daniel Moreno Soto, Biol. (10) and Walter D. Cardona Maya PhD (1) \\ Reproduction Group, School of Medicine, University of Antioquia, Medellín, Colombia
}

To the Editor-The coronavirus disease 2019 (COVID-19) pandemic has killed hundreds of thousands, has infected millions,

\footnotetext{
Author for correspondence: Walter D. Cardona Maya, E-mail: wdario.cardona@udea. edu.co

Cite this article: Moreno Soto D and Cardona Maya WD. (2021). Forewarned but not forearmed: The risk of science driven by immediacy. Infection Control \& Hospital Epidemiology, 42: 791-793, https://doi.org/10.1017/ice.2020.350
}

and has ravaged the economy worldwide in $<6$ months. The full implications of this crisis are still unfathomable and might span years or even decades. The virus has bewildered everybody, raising the feeling that we were not prepared and even mining the trust of many sectors of the population on the capability of science to overcome the crisis. Nonetheless, to say that what happened was not

(C) 2020 by The Society for Healthcare Epidemiology of America. All rights reserved. This is an Open Access article, distributed under the terms of the Creative Commons Attribution licence (http://creativecommons.org/licenses/by/4.0/), which permits unrestricted re-use, distribution, and reproduction in any medium, provided the original work is properly cited. 
(a)
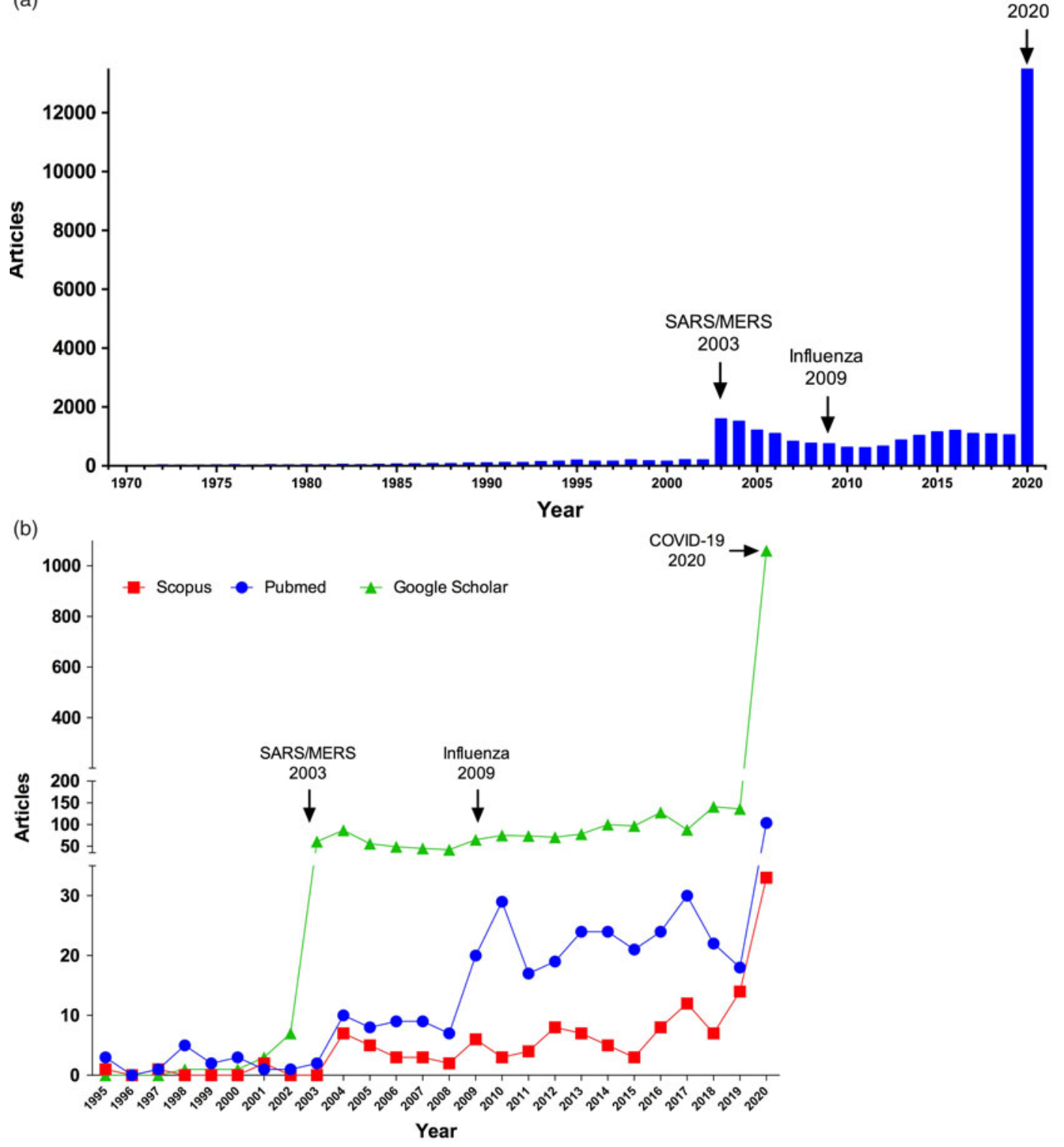

Fig. 1. (a) Articles published containing the words SARS-CoV-2, SARS-CoV-1, COVID-19, SARS, or MERS. (b) Peaks of publications in 2003 and 2009 are evident, probably related to the outbreaks of SARS and influenza, respectively. The same pattern is observed in publications related to N95 masks from 1995 to 2020.

predictable and that we could not have done better would not only be inaccurate but also accessory by negligence.

In the history of humankind, we have never been so technologically capable of dealing with a pandemic, yet we have performed relatively poorly in many aspects. Setting aside the social, political, and economical nuances that have hindered the best-case scenario, as well as the intrinsic uncertainty associated with an event of this magnitude, the untimely commitment to long-known research necessities has been a decisive factor in the inadequate response.

In far too many cases, the trend has been to massively research ongoing threats, often fueled by politically driven funding, to later quit abruptly after the peril has passed or when the media interest has diminished. The research was thus rendered incomplete and did not yield any real solutions, even when results were very close. This behavior has left us in no significantly better condition to cope with the recurrence of similar situations despite the laudable efforts of scientists during times of crisis.

Over the past 50 years, research in PubMed (ie, using search terms, SARS-CoV-2 or SARS-CoV-1 or COVID-19 or SARS or MERS or coronavirus on June 15,2020) regarding the Coronaviridae family has peaked during outbreaks and has flattened between them (Fig. 1a). This dynamic is also evident in research regarding technologies that are crucial for the management of epidemics, such as personal protective equipment (PPE). Medical masks and facepiece respirators, such as N95, are intended for single use. However, during outbreaks, shortages are inevitable and single use is overlooked. In recent decades, alternatives for reusable masks or safe disinfection protocols have been explored only in times of pressing need (Fig. 1b), and clearly, we were not well prepared in this aspect when COVID-19 broke out. 
The severe acute respiratory syndrome (SARS) 2002-2004 epidemic could have served us better had we attended carefully to the lessons of that time. The evidence that presaged our current reality was recklessly and inexcusably overlooked. For more than a decade, the world disregarded evidence showing that wildlife markets in China, along with the high genetic recombination rates of coronaviruses, comprised an environment ripe for another zoonotic outbreak. ${ }^{1}$ We also missed a chance to achieve the only definitive solution to the pandemic when several promising SARS vaccines, which had undergone preclinical trials, were thwarted by a lack of further funding. ${ }^{2}$ Although SARS and SARS-CoV-2 are different viruses, their genetic closeness and similarity in the molecular mechanism of infection would have saved valuable time in the proper development of a SARS-CoV-2 vaccine. Instead, we are now rushing phase 1 clinical trials without preclinical or animal models. ${ }^{2}$

This pattern of scattered research might be a trademark of the way in which science has operated in contemporary society, but the vulnerability derived from allowing it to persist this way is unreasonable. Newton's exceptionally hackneyed quote, "If I have seen further it is by standing on the shoulders of giants," superbly conveys the notion of science being a cooperative effort, and we must always remember that these shoulders are often broadly spread across time. Public funding, as well as the overall mentality underlying research, cannot be steered toward achieving results in the short term or, otherwise, not achieving any results at all. Some processes, such as new PPE technologies and vaccines, must be understood and acknowledged as intrinsically time-consuming and must be continuously supported outside times of critical necessity. As evidence during the COVID-19 crisis shows, the real-time capacity to find solutions is insufficient and the price that we must pay for missed opportunities it is too high.

\section{Acknowledgments}

Financial support. No financial support was provided relevant to this article.

Conflicts of interest. All authors report no conflicts of interest relevant to this article.

\section{References}

1. Cheng VCC, Lau SKP, Woo PCY, Kwok YY. Severe acute respiratory syndrome coronavirus as an agent of emerging and reemerging infection. Clin Microbiol Rev 2007;20:660-694.

2. Subbarao K. SARS-CoV-2: a new song recalls an old melody. Cell Host Microbe 2020;27:692-694.

\title{
Occupational exposure to varicella zoster in a tertiary-care healthcare setting
}

\author{
Zachary A. Yetmar MD ${ }^{1}$ (1), Debra K. Apenhorst MAN, RN², Melanie D. Swift MD, MPH ${ }^{3}$, Priya Sampathkumar MD ${ }^{1}$ \\ and Elena Beam MD ${ }^{1}$ \\ ${ }^{1}$ Division of Infectious Diseases, Mayo Clinic, Rochester, Minnesota, ${ }^{2}$ Infection Prevention and Control, Mayo Clinic, Rochester, Minnesota and ${ }^{3}$ Division of \\ Preventive, Occupational and Aerospace Medicine, Mayo Clinic, Rochester, Minnesota
}

To The Editor-Varicella zoster virus (VZV) reactivation is a common complication of a weakened immune system, which can occur due to advanced age or various immunocompromising conditions. The VZV incidence in the general population is 4.82 cases per 1,000 person years, ${ }^{1}$ but this rate increases in populations with predisposing conditions. Solid-organ transplant recipients are estimated to have a VZV incidence of 22.2 cases per 1,000 patient years, with heart transplant recipients having the highest organ-specific incidence of 40.0 per 1,000 patient years. ${ }^{2}$ Hematopoietic stem cell transplant recipients have reported incidence rates of 43-60 cases per 1,000 person years. ${ }^{1,3}$ With high incidence and subsequent healthcare utilization in these populations, there is an important need to prevent occupational exposure to VZV.

Recommendations for isolation precautions differ by extent of VZV involvement and immunocompromised status. For immunocompetent patients, the Centers for Disease Control and Prevention recommend contact isolation for localized VZV and concurrent airborne and contact isolation for disseminated VZV. ${ }^{4}$ In immunocompromised patients with apparent localized disease, contact and

Author for correspondence: Zachary A. Yetmar, E-mail: yetmar.zachary@mayo.edu Cite this article: Yetmar ZA, et al. (2021). Occupational exposure to varicella zoster in a tertiary-care healthcare setting. Infection Control \& Hospital Epidemiology, 42: 793-795, https://doi.org/10.1017/ice.2020.351 airborne isolation are recommended until disseminated disease has been ruled out. However, it is not known whether instituting airborne isolation in this population reduces occupational exposure to VZV. At our facility, we only institute contact isolation in cases of localized $\mathrm{VZV}$, regardless of immunocompromised status. We reviewed cases of disseminated VZV to evaluate whether this change in policy increased the likelihood of occupational exposure.

We performed a retrospective, descriptive review of occupational exposure investigations related to VZV. We included patients from January 2016 through December 2018 and excluded those with primary chicken pox. Demographic and clinical data were abstracted from the electronic medical record. Records were evaluated to determine whether the exposure was due to a delay in airborne precaution initiation or a progression of localized disease at presentation to disseminated VZV.

In total, 23 patients met our inclusion and exclusion criteria; 12 patients (52.2\%) were female, with a median age of 64 years (interquartile range, $57-70.5$ years). Also, 20 patients (87.0\%) had an immunocompromising condition. This cohort included 8 patients (34.8\%) with a hematologic disorder or malignancy, 4 patients (17.4\%) with a solid-organ malignancy, 3 patients $(13.0 \%)$ with a bone marrow transplant, 3 patients $(13.0 \%)$ receiving immunosuppressing medication, and 1 patient $(4.3 \%)$ with a solid-organ 